CERN-EP/81-145

10 November 1981

\title{
Live target for lifetime measurements of charmed particles and related signal processing
}

\author{
G. Bellini*+), P. D'Angelo $^{+1}$, P.F. Manfredi**+) E. Meroni*t), L. Moroni ${ }^{+1}$ \\ C. Palazzi Cerrina ${ }^{++}$, F. Ragusa ${ }^{++}$and S. Sala ${ }^{+1}$ \\ Istituto Nazionale di Fisica Nucleare, \\ Sezione di Milano, Milano, Italy.
}

\begin{abstract}
A telescope of silicon detectors has been designed as a live target for lifetime measurements of charmed particles. Along with the target, a low-noise data acquisition system has been developed, suitable for operation at counting rates beyond $10^{6}$ particles per second. The target, successfully employed in a high-energy experiment at the CERN Super Proton Synchrotron, is described in this paper, which also provides detailed discussion about the design criteria and the performance of the signal processing adopted to cope with the experiment constraints.
\end{abstract}

(Submitted to Nuclear Instruments and Methods)

+) Visitor at CERN, Geneva, Switzerland.

*) Istituto di Fisica dell’Università, Milano, Italy.

**) Istituto di Elettronica e Comunicazioni Elettriche, Università di Pavia, Italy. 


\section{INTRODUCTION}

A powerful way of investigating high-energy reactions is to make them occur in a detecting device, for instance in a silicon target located in the accelerator beam. The target in this case is called live, to account for the fact that it also performs a detecting function").

To reduce the uncertainty in the position at which the reaction takes place, a target of a given amount of material is realized by aligning several adequately thin silicon detectors $\mathrm{s}^{2}$.

There are several fundamental reasons for employing live targets: the possibility of identifying the interaction point with high accuracy; the clear signature that live targets provide for incoherent events where the nucleus break-up is followed by emission of slow protons; and the fact that they allow accurate energy evaluations of nuclear coherent recoil and supply information about the charged multiplicity of the reaction $^{3)}$. Live targets can therefore be considered as high-resolution vertex detectors.

In the design of a live target, a parameter that is difficult to assess is the thickness of silicon detectors, as will be shown below. The recoil energy is evaluated by subtracting from the total energy released in the detector the contribution due to minimum-ionizing, incoming and outgoing particles. The accuracy of the result is affected by the fact that the interaction point within the detector is unknown, and therefore the energy released by minimum-ionizing particles can be estimated only in an approximate way ${ }^{4}$. Such an inaccuracy term is proportional to the detector thickness, which limits the spatial resolution in the vertex reconstruction. A reduction in the detector thickness, however, results in a larger detector capacitance, i.e. in enhanced noise contribution from the series generator in the head amplifier.

Live targets can be successfully employed in measuring lifetimes between $10^{-13}$ and $10^{-12} \mathrm{~s}$, this being the range of interest for charmed particles at the energies of presently available accelerators. With $\mathrm{TeV}$ accelerators, the lower limit can be further reduced by virtue of the relativistic $\gamma$ factor.

Lifetime measurements can be performed with a telescope of silicon detectors located in a beam, and by identifying production and decay points from nucleus recoil and from variations in the energy released in the detectors. The basic advantage of this device over nuclear emulsions is that it can accumulate large amounts of statistics. In order to exploit it fully; however, it is necessary to associate the telescope with a data-acquisition system capable of operating on high-intensity beams.

The live target which will be described in this paper was designed for lifetime measurements of charmed particles in a photoproduction experiment at the CERN Super Proton Synchrotron (SPS) (FRAMM-NA1 experiment). The target was associated with a forward spectrometer, which provides the reconstruction of secondary particles ${ }^{5}$.

The topology of the accepted reactions was restricted to coherent photoproduction of a pair of charmed mesons. This constraint increases the efficiency of the target in identifying production and decay points. According to the results of a Monte Carlo simulation ${ }^{6}$, the thickness of the detectors and their distances in the telescope were initially optimized for lifetimes of $5 \times 10^{-13} \mathrm{~s}$, for energies of incident photons corresponding to a beam spectrum extending up to $150 \mathrm{GeV}$.

To make the target more flexible so as to suit lifetime measurements in different ranges; the possibility of changing the spacing between the detectors and of replacing some detectors with others of different thicknesses was provided for.

\section{THE FRAMM LIVE TARGET}

The live target employed in the FRAMM experiment consists of a telescope $T_{1}$ of 30 silicon detectors followed by a telescope $T_{2}$ made of 10 detectors. Telescope $T_{1}$, described by figs. $1 \mathrm{a}$ and $1 \mathrm{~b}$, was designed by the Milan group along with ENERTEC, who manufactured it using an ion implantation technique; whilst $T_{2}$ was developed by Pisa University using the surface barrier approach").

The detectors of both telescopes are circular in shape with a diameter of $14 \mathrm{~mm}$ and a thickness of $300 \mu \mathrm{m}$, and are stacked approximately $150 \mu \mathrm{m}$ apart from each other. The capacitance presented by each detector in telescope $T_{1}$ is about $300 \mathrm{pF}$, a value considerably!larger than the $80 \mathrm{pF}$ expected for an isolated silicon layer of the same area and thickness. There are two reasons for the increased capacitance. First, there is the contribution of the $150 \mu \mathrm{m}$ thick air capacitor introduced by the presence of the adjacent detector. 
Secondly, the close distance gives, as a side effect, an increased value of the edge capacitor which is determined by the collecting electrode and the undepleted silicon regions. The assembling technique adopted in telescope $T_{2}$ (ref. 7) results in a slightly reduced value of the capacitance appearing between the terminals of every detector, but has the limitation of a larger and less stable leakage current. The leakage currents of $T_{1}$ and $T_{2}$ were monitored during the experiment and kept at reasonably low values, of the order of $0.1 \mu \mathrm{A}$ and $1 \mu \mathrm{A}$, respectively, by forced-air ventilation which removed from the target the heat generated by surrounding devices.

In the actual experimental conditions, a high-intensity photon beam hits the target. The number of electron pairs created by photon materialization grows almost linearly along the detector, as does the counting rate in the detectors, approaching about $10^{6}$.particles per second in the last layers. To cope with this non-uniform rate, without needlessly sacrificing the signal-to-noise ratio in the acquisition channels associated with detectors working at lower charged-particle intensity, two different signal processors were designed. The first group of 20 detectors is, accordingly, served by trapezoidal shapers with $0.5 \mu$ s pulse width, featuring higher-energy resolution. Signals from the second group of 20 detectors are instead processed by fast triangular shapers with $0.1 \mu \mathrm{s}$ pulse width and superior counting rate performances. In either case the most important limitation in signal-to-noise ratio is related to the large detector capacitance. Effort was therefore directed towards designing head amplifiers with a low slope in the noise dependence of the detector capacitance.

To understand the difficulties connected with this type of low-noise data acquisition, it has to be borne in mind that in a $300 \mu \mathrm{m}$ silicon detector a minimum-ionizing particle releases only about $80 \mathrm{keV}$.

\section{DATA ACQUISITION FROM THE FRAMM TARGET}

The block diagram of the data acquisition system developed for the FRAMM target is shown in fig. 2 . Each detector is associated with an acquisition channel consisting of a linear processor followed by an analog-to-digital converter (ADC). Linear processing of detector signals is performed by a low-noise head amplifier and by either a $0.5 \mu$ s trapezoidal filter or a $0.1 \mu$ s triangular filter. After shaping, restoration is employed to reduce base-line fluctuations at high counting rates. The linear section of the data-acquisition channels is of a time-invariant nature and therefore all the detector signals are processed in the same way. Most of them, however, are prevented from reaching the ADCs by linear gates that are normally closed. The gates open only for those events for which (the trigger requirements being met) the data-acquisition command is generated. The selected signals are digitized by the ADCs. Two different types of commercial ADCs were employed to sort the signals from the linear processors. Signals delivered by the slower shapers were digitized by peak-sensing ADCs, whilst charge-sensing units were adopted for those coming from the $0.1 \mu \mathrm{s}$ shapers. The latter solution, although not perfectly suitable for the purpose of the actual measurement (which would require peak sensing), was made compulsory by the lack of commercial ADCs with an adequately fast peak-stretcher.

The events, once digitized and made available on the CAMAC dataway, are transferred to a PDP11/34 computer which implements data collection and on-line event monitoring. On-line monitoring includes the displaying of a pulse-height spectrum from each detector during data acquisition, and event-by-event amplitude patterns in the telescope.

The events selected by the data-acquisition command are labelled with a pile-up mark and discarded off-line if they are preceded or followed by other signals within the shaping time of the processors. To monitor pile-up.occurrence; fast timing signals are derived from the 10th, 20th, and 30th detector, and these trigger three thireshold discriminators. Two outputs are taken from each discriminator. One of them is kept in time with the data-acquisition command, and one is delayed by an interval equal to the desired inspection time: Both outputs are used as the STOP signals of a time-to-digital converter (TDC), whose START is triggered by the data-acquisition command. In this way any signal occurring before or after the accepted event, and which might disturb it, stops the TDC, thus generating the pile-up label.

The stability, linearity, and resolution of the data-acquisition channels are regularly monitored using a calibration procedure, according to which three reference charges are injected at the input of the head 
amplifiers, and the resulting signals are processed, digitized, and stored. The reference charges are obtained by applying, across accurately known injection capacitors, the artificial voltage signals taken from a computer-controlled attenuator. These charges simulate energies, released in silicon, of $114.5,229$, and $458 \mathrm{keV}$ per picofarad of injection capacitance. The calibration procedure takes place in the beam-off intervals between two consecutive bursts.

\section{THE LINEAR PROCESSORS}

As already pointed out, two different types of linear processors were developed to cope with the different counting-rate requirements of the first and the second group of detectors. The processor with lower counting-rate characteristics consists of a charge-sensitive head amplifier, a trapezoidal filter, and a time-variant base-line restorer. The processor with advanced counting-rate behaviour employs a fast charge-sensitive head amplifier, followed by a triangular shaper and a highly asymmetric base-line restorer ${ }^{8)}$.

The responses of processors of either type to a narrow calibration pulse injected at the input of their head amplifiers are shown in fig. 3 .

Trapezoidal and triangular shapings are implemented by delay-line circuits, and they were chosen because they allow a particularly fast tail-free recovery at the end of the pulse.

The charge-sensitive head amplifiers for either type of processor were designed to suit the actual application, which involves large detector capacitances and short processing times, these being determined by the base-width of the trapezoidal pulse and of the triangular pulse. In the operating condition described, the rise-time of the head amplifier, determined by the detector capacitance and by the "cold resistance" presented by the charge-sensitive loop, is not negligible with respect to the processing time ${ }^{9)}$.

Consequently, the amplitude of the shaped pulses, either trapezoidal or triangular, will depend on the rise-time of the head amplifiers. To avoid inaccuracies and instabilities in the final amplitude, the rise-time of the head amplifiers was stabilized by employing charge-sensitive loops with feedback-controlled cold resistance ${ }^{10)}$.

Good energy resolution at large detector capacitance is obtained by utilizing, as the input stage of the charge-sensitive loops, two high $g_{\mathrm{m}}$ field-effect transistors (FETs) (type Teledyne-Cristalonics 2N6550), or alternatively two selected JFETs (type Texas Instruments SFS 8558). The detector capacitance dependence of noise line-width referred to the input for both types of processor is shown in fig. 4 . These results were obtained with the base-line restorers excluded.

Head amplifiers and trapezoidal shapers are based upon already discussed circuit configurations ${ }^{11)}$ Here, attention will be restricted to the $0.1 \mu$ s triangular processor. Its detailed circuit diagram is shown in fig. 5. The input signal, which is delivered by the head ampliffer with a slowly decaying exponential tail, is first amplified by two fast feedback stages, transistors $T_{1}, T_{2}, T_{3}, T_{4}, T_{5}$ and $T_{6}, T_{7}, T_{8}, T_{9}, T_{10}$. After amplification it is passed to a cable terminated at the transmitting end and short-circuited at the receiving end: The resulting current in the outer conductor of the cable has a bipolar shape and its duration is twice the cable delay. As shown by fig. 5 , this current is injected into the virtual ground of an operational integrator $\left(J, T_{11}, T_{12}\right)$ which provides a triangular signal at the output. After base-line restoration $\left(T_{13}, T_{14}, T_{15}, T_{16}\right)$, the signal is sent through a buffer $\left(T_{17}, T_{18}, T_{19}, T_{20}\right)$ to a fast linear gate employing diode bridges in a series-parallel configuration, and thence to the output amplifier.

The advantage of the triangular shaper of fig. 5 over an $R C-C R$ filter with the same peaking time is evident from fig. 6 . The triangular shaper recovers to zero after the peak in a much faster way, thus reducing the pulse-on-tail pile-up effects àt high counting rates.

\section{PERFORMANCE AND LIMITATIONS OF THE SYSTEM}

The FRAMM data-acquisition system was designed to process events with multiplicities between 1 and 12 , and the upper limit of the linear range was accordingly set at about $1.2 \mathrm{MeV}$. Events within the linear range are digitized with an 11-bit resolution by either type of $\mathrm{ADC}$, peak or charge-sensing. 
The long-term stability of an acquisition channel is described by fig. 7, where the drift in the centroid position of one of the artificial calibration lines is represented. The values of fig. 7 were measured at intervals of few hours during a total data-taking period lasting 150 hours. Despite the relatively large temperature variations occurring in the beam hall, where the head amplifiers were located, very few points were found outside the $\pm 1 \%$ band, most of them falling within the $\pm 0.5 \%$ limits.

The characteristics of energy resolution of the acquisition channels can be inferred from fig. 8 . The spectral lines of fig. 8 are the reconstructed Landau distributions of one, two, or four minimum ionizing particles obtained with a processor of the $0.5 \mu$ s trapezoidal type (fig. 8a) and with a processor of the $0.1 \mu \mathrm{s}$ triangular type (fig. 8b).

The behaviour of both types of processor at high counting rates is described by the spectra of fig. 9 . Figure 9 represents the Landau distributions of single minimum-ionizing particles taken at three different counting rates. To obtain the data of fig. 9 , the amplification of the processors was increased with respect to the ordinary value employed under experimental conditions, in order to have the spectra displayed on an adequately large number of $\mathrm{ADC}$ bins.

As shown by fig. $9 \mathrm{a}$, the $0.5 \mu$ s trapezoidal processor exhibits a small change in the line shape when the rate is raised from $3.4 \times 10^{4} \mathrm{pps}$ to $2.5 \times 10^{5} \mathrm{pps}$, but introduces a sensible distortion at $1.7 \times 10^{6} \mathrm{pps}$. The Landau centroid has $1.4 \%$ shift between $3.5 \times 10^{4}$ and $2.5 \times 10^{5} \mathrm{pps}$, but the shift approaches $10 \%$ when the rate is increased to the highest value. However, with the actual experimental conditions, and with a photon beam hitting the target, the rates in the first group of detectors are of the order of $10^{5} \mathrm{pps}$ and the behaviour of the $0.5 \mu \mathrm{s}$. processor is fully satisfactory. According to the results of fig. $10 \mathrm{~b}$, the $0.1 \mu$ s shaper operates properly up to the highest rate available under test conditions. There is no fundamental change in the spectrum shape, the noticeable effect being an increase of the counts in the Landau tail. As the rate is raised from $3 \times 10^{4}$ to $1.7 \times 10^{6}$ pps, the centroid shift is less than $3 \%$ and the r.m.s. width change is about $7 \%$.

The good energy resolution of the system, under the actual experimental conditions, is shown in fig. 10 . Also shown are the background peak and the peak of electrons pairs created by materializing photons, for detectors in different positions along the telescope. The latter peak increases, whilst the former decreases from the first to the last detector. In the last detector the line due to four charged particles begins to emerge from the background.

The FRAMM target was employed to take data with a spectrometer which provides measurements of charged particles and neutral $\pi$ mesons. About 1.8 million triggers were collected, having the following requirements: at least two hadrons and one photon in the forward spectrometer ( $\pm 90 \mathrm{mrad}$ for charged particles and $\pm 30^{\circ}$ for the photons) and nothing outside the solid angle. The silicon target did not have any part in the trigger definition ${ }^{12,13)}$.

The analysis started with the associated production of charged $\mathrm{D}$. The target information has been used at two different stages. In the first one, nuclear coherence. requirements and cuts in total multiplicity led to a reduction of a factor of 10 to 100 in the number of events to be processed. Events selected in this way are analysed in the forward spectrometer to identify charm production candidates. As a second step, the target information is used for the identified candidates, looking for production decay (or decays) sequences. Two examples of the final selected sample are shown in figs. $11 \mathrm{a}$ and $11 \mathrm{~b}$, where the pulse-height patterns in the target detectors are presented along with the event reconstruction made by the forward spectrometer. Diagram (c) of fig. 11 provides an example of the incoherent production of a pair of D's: one of the D's decays inside the production target. The device described presents a limitation when both $D$ 's decay into the same number of charged prongs. In such a case there is no way of relating the path measured in the target with the relativistic factors obtained from the spectrometer. The average value of $\gamma$ can be used assuming that

$$
\gamma_{\mathrm{D}}=\gamma_{\overline{\mathrm{D}}}=\frac{E_{\mathrm{tot}}}{M_{\mathrm{D}}+M_{\overline{\mathrm{D}}}} .
$$

Main sources' of background in the target operation are secondary interactions and $\gamma$ materialization. Both sources can be avoided if the target is combined with a spectrometer which measures and recognizes the particles, and if the electron pairs' are produced by secondary $\pi^{0 \%}$ s. The forward spectrometer is, however, of 
no use against conversion of double-bremsstrahlung soft photons produced in the primary beam and coincident in time with an energetic incident photon. In most cases these photons give a large signal in only one detector, thus simulating a nucleus recoil.

\section{CONCLUDING REMARKS}

A target of 40 silicon detectors has been realized for measuring the lifetime of charmed particles. Data acquisition from the target is performed by a low-noise system, which was designed bearing in mind the stringent requirements dictated by the experiment. The data-acquisition system has to cope with the low energy released in the detectors, with the large detector capacitance, and with very high counting rates. Improvements are being planned with a view to future extensions of the described technique in the domain of lifetime measurements. They aim basically at reducing the capacitance of the detectors and increasing the counting rate capabilities of the acquisition channels. Detector sectioning, to reduce the surface and therefore the capacitance presented at the input of every head amplifier, is now being investigated, along with alternative detector manufacturing processes and assembling techniques. In the meantime, new ideas in the field of analogue processing which should lead to a shorter signal shaping without appreciable deterioration in signal-to-noise ratio are also being tested. 


\section{References}

[1] G. Bellini et al., Nucl. Instrum. Methods 107 (1973) 85.

[2] G. Bellini et al., Active targets as a tool to study short-range interaction phenomena in high-energy experiments. Proc. of the Meeting on the Miniaturization of High-Energy Physics Detectors, Pisa, September 1980 (to be published).

[3] E. Albini et al., Use of nuclear targets in FRAMM experiment. Lifetime measurements on charmed mesons; NA1/7917, Milan (1979).

[4] G. Bellini et al., Live targets to measure heavy-particle short lifetimes and to trigger on charm particles, Contribution to the Workshop on Fixed-Target-Physics, FNAL, Batavia, 1980 (FNAL, Batavia, 1980).

[5] Frascati-Milano-Pisa-Roma-Trieste Collaboration, Photoproduction of vector and scalar bosons, CERN/SPSC/79-112 SPSC/M 203(1979).

[6] E. Albini et al, A live target for measuring the lifetime of charmed particles, Contributed paper No. 0795 to the "20 $20^{\text {th }}$ Int: Conf. on High-Energy Physics"; Madison, 1980.

[7] S.R.Amendolia et ali, Nucl. Instrum. Methods, 176 (1980) 457.

[8] L.O. Johnson, IEEE Trans. Nucl. Sci. NS 26 (1979) 474.

[9] P.F. Manfredi, Electronics for silicon detectors in high-energy experiments. Proc. of the Meeting on the Miniaturization of High-Energy Physics Detectors, Pisa, September 1980 (to be published).

[10] E. Gatti and P.F. Manfredi, Low-noise current preamplifiers for large capacitance semiconductor detectors and high counting rates, Proc. 2nd Ispra Nuclear Electronics Symposium (Publication EUR 5370e, 1975), p. 33.

[11] M. Cisotti et al., Nucl. Instrum. Methods 159(1979) 235.

[12] Frascati-Milano-Roma-Trieste Collaboration, Evidence for $\mathrm{DD}$ diffractive photoproduction and future plans. Status report of NA 1 experiment, CERN/SPSC/80-85 SPSC/M247(1980).

[13] Frascati-Milano-Pisa-Roma-Trieste Collaboration, Charmed meson pair coherent photoproduction off active silicon target. Paper submitted to the European Physical Society Int. Conf. on High-Energy Physics, Lisbon, 1981. 


\section{FIGURE CAPTIONS}

Fig. 1: FRAMM active target:

a) front view of telescope $T_{1}$,

b) lateral view of the same telescope.

Fig. 2: Block diagram of the acquisition system developed for the FRAMM target.

Fig. 3: Response of trapezoidal and triangular shapes to an artificial current pulse simulating the detector signal:

a) $0.1 \mu \mathrm{s} \mathrm{trapezoidal} \mathrm{shaper,}$

b) $0.5 \mu$ s triangular shaper.

Fig. 4: Noise line-width, FWHM referred to the input, as a function of detector capacitance $C_{\mathrm{D}}$.

Measurements were made without base-line restorers.

Fig. 5: Detailed circuit diagram of the $0.1 \mu$ s triangular processor.

Fig. 6: Comparison between the $0.1 \mu$ s base-width triangular and the RC-CR shaped signal of equal peaking times.

Fig. 7: Long-term drift of one of the acquisition channels.

The relative change in centroid position of one of the calibration lines is monitored at intervals of a few hours, for a total data taking period of 150 hours.

Fig. 8: Landau distributions of one, two, and three minimum-ionizing particles crossing a $300 \mu \mathrm{m}$ thick detector, obtained with the $0.5 \mu$ s processor (a) and with the $0.1 \mu$ s processor (b).

Fig. 9: Landau distribution of single minimum-ionizing particles at three different counting rates:
a) $0.5 \mu$ s trapezoidal processor

b) $0.1 \mu$ s triangular processor.

Fig. 10: Spectra taken from five equally spaced points along a telescope in the actual experiment conditions; that is, with a photon beam impinging on the target.

Fig. 11: Examples of three different events detected by the target and compared with the spectrometer outcome. The signal amplitudes from the silicon detectors are expressed in units of single minimum-ionizing particle. 

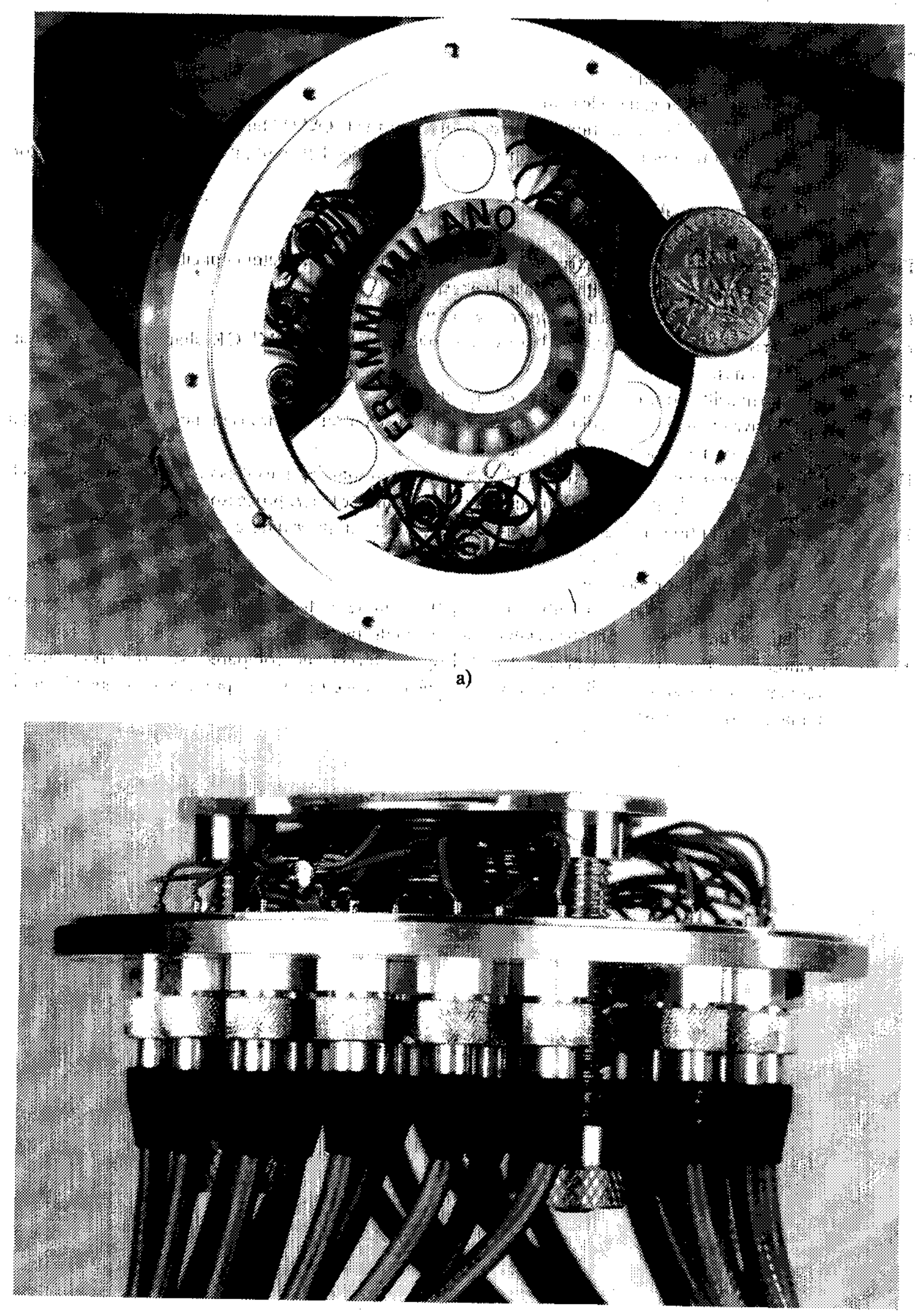

b)

Fig. 1 


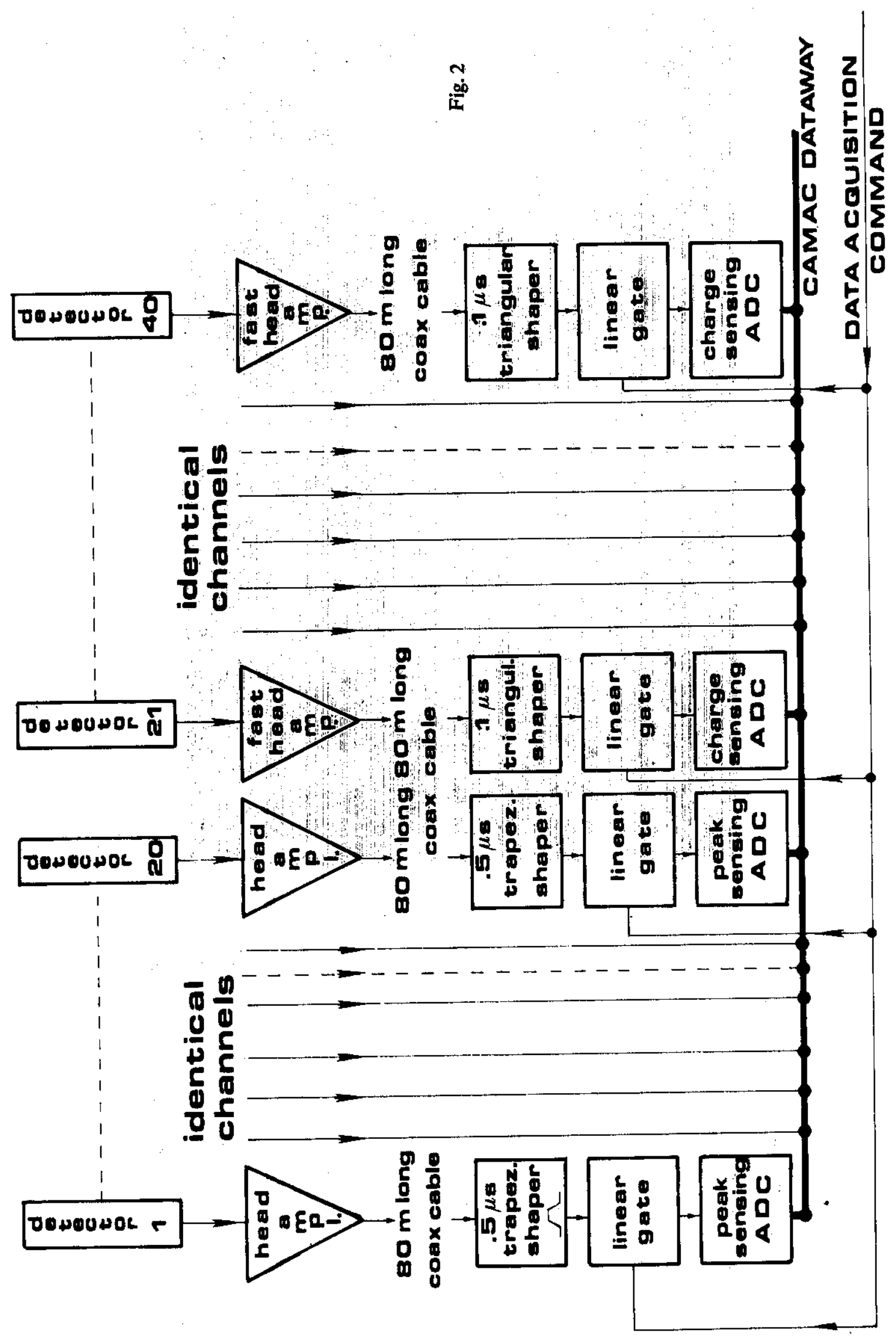




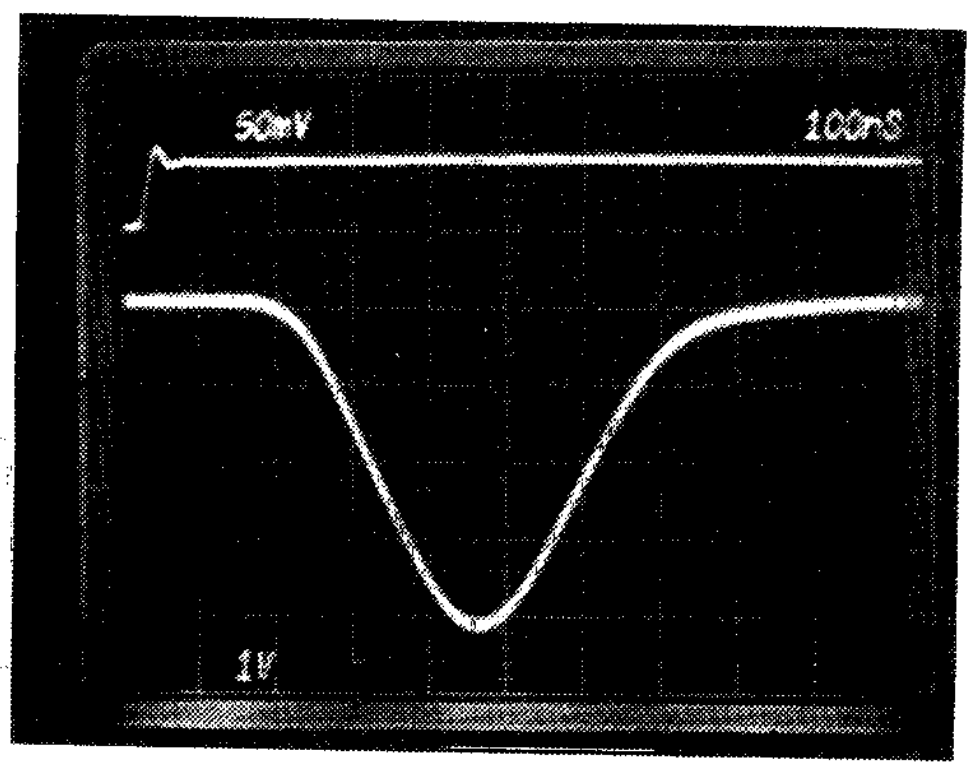

a)

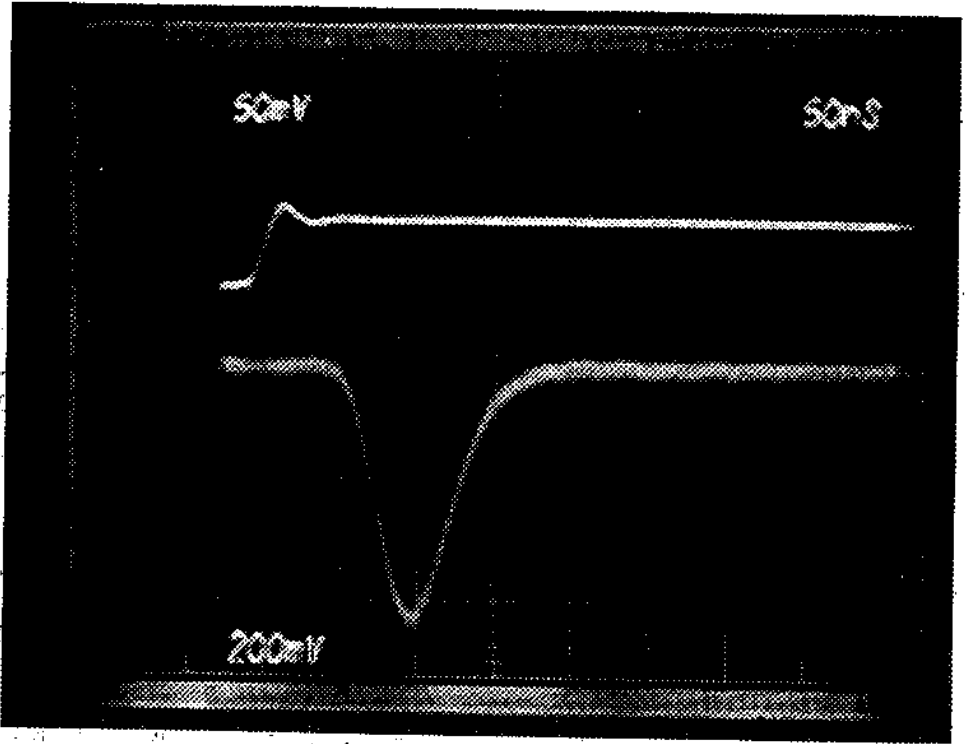

b)

Fig. 3

Resolution FWHM

80- keV Si equivalent

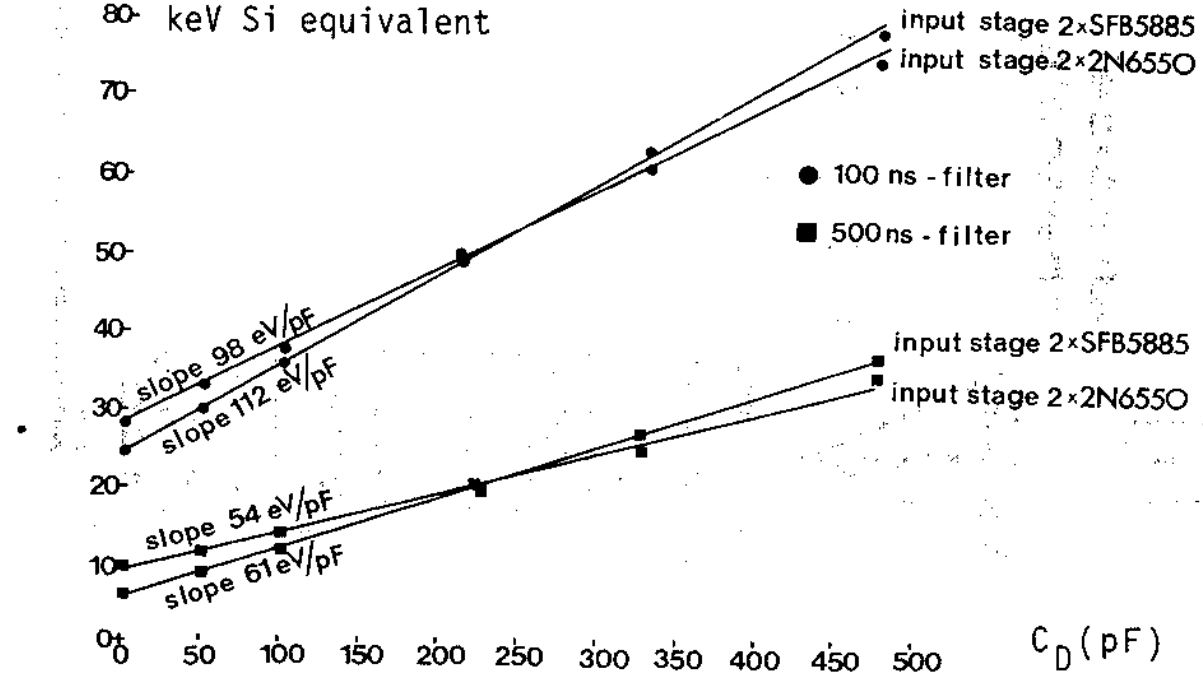

Fig. 4 


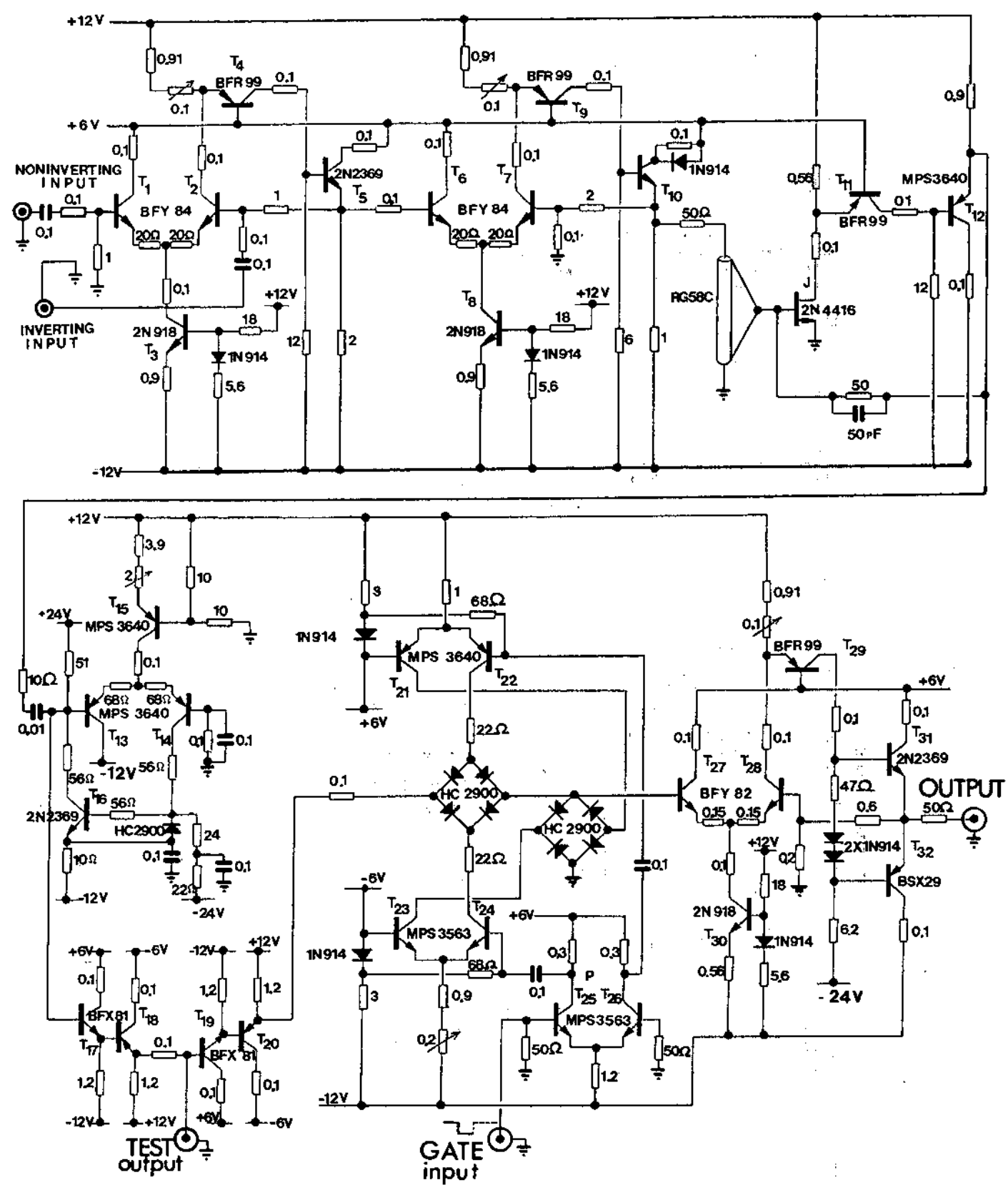

Fig. 5

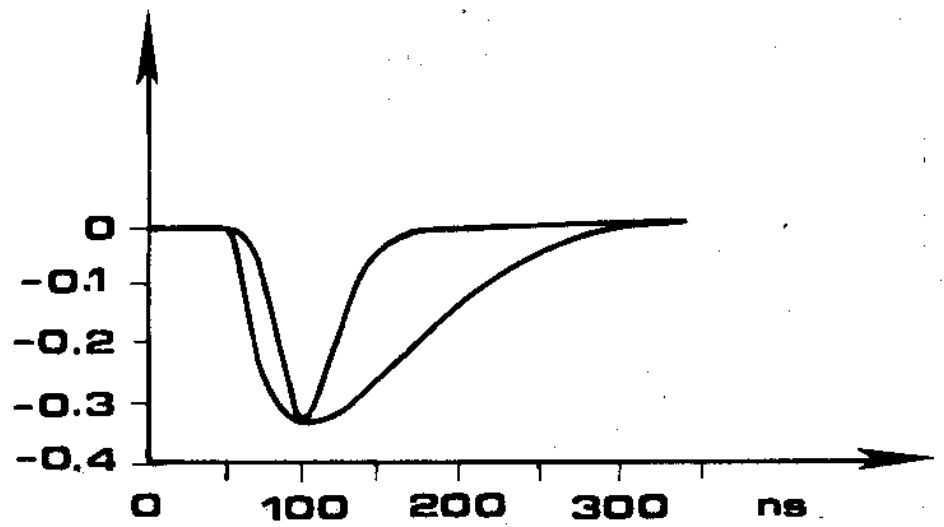

Fig. 6 


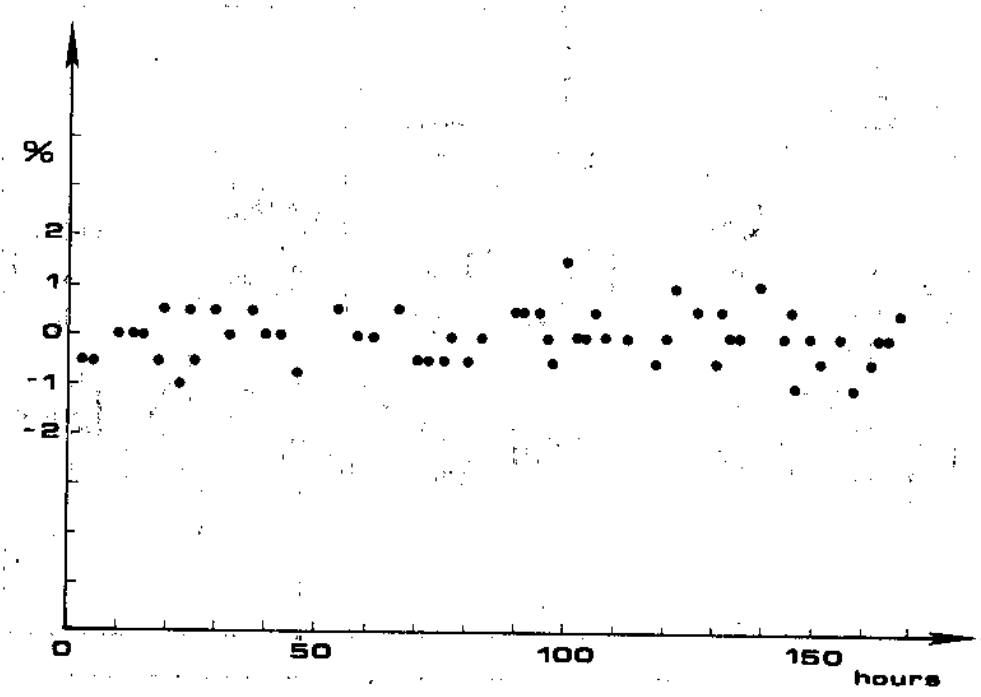

Fig. 7
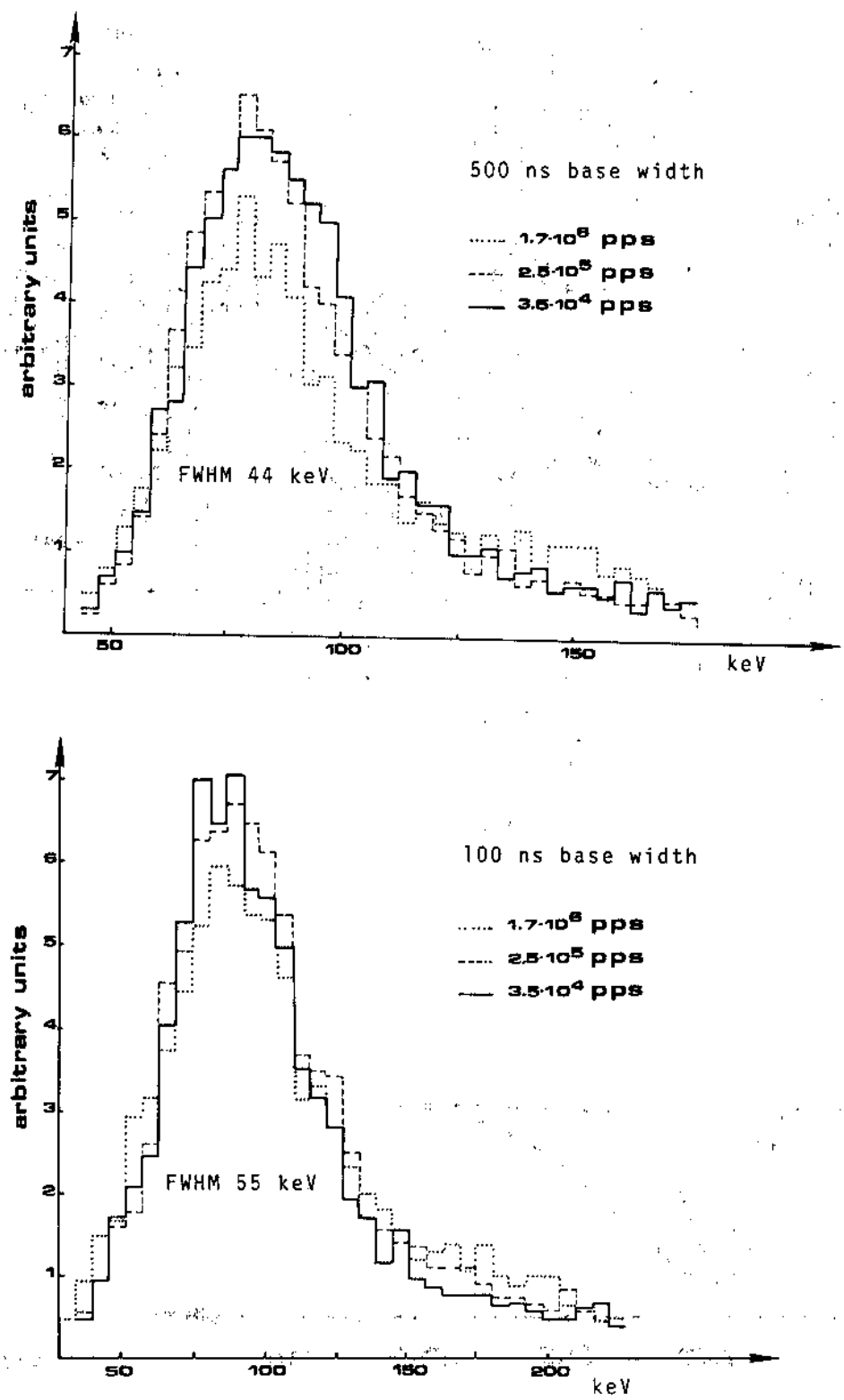

a)

b)

Fig. 8 


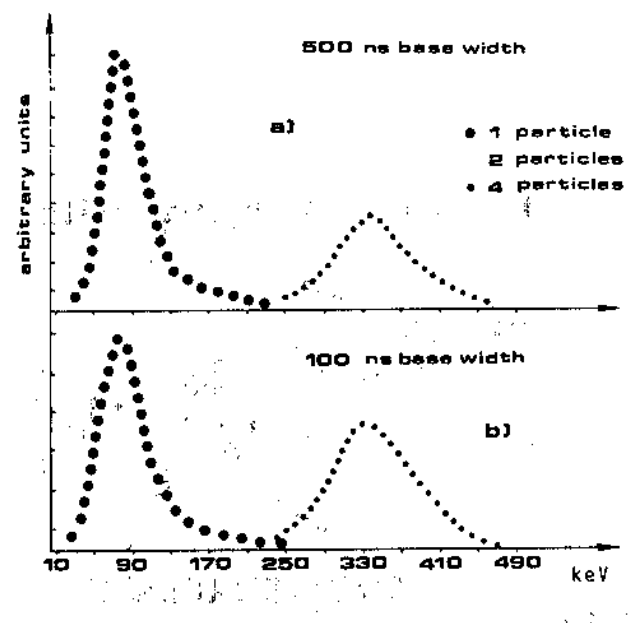

Fig. 9

RLIH :750 Q9-FEE-9Q $13: 22: 45$
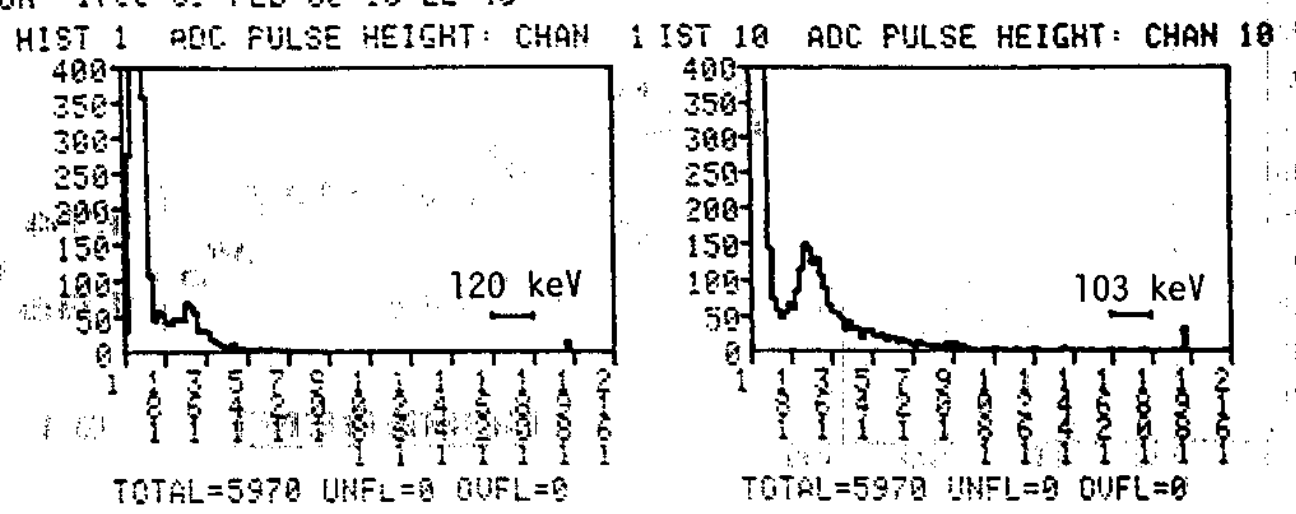

RuW 1750 O3-FEE-80 $13: 22: 46$

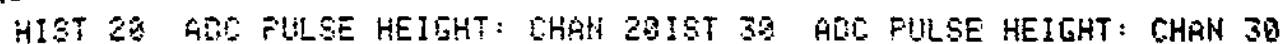
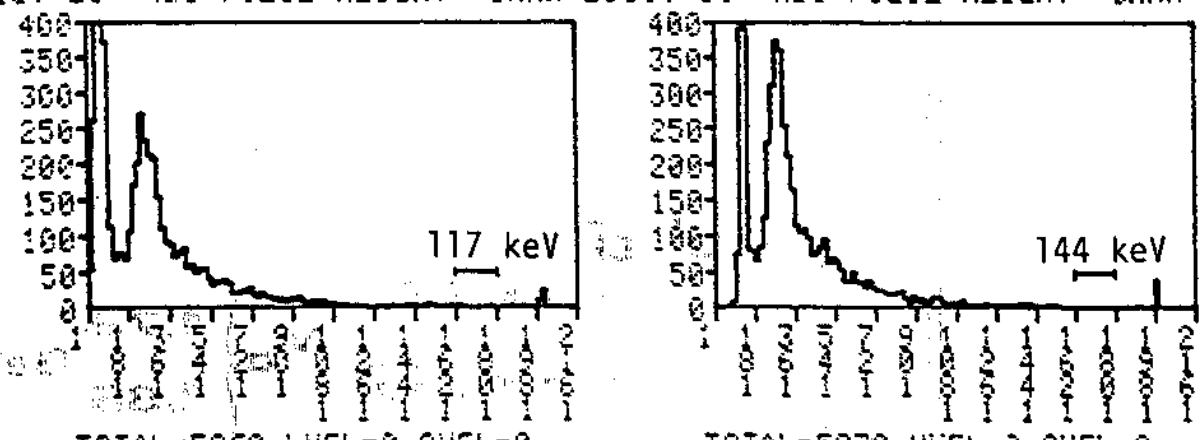

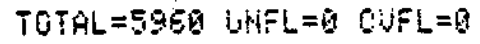

TOTA่L=5978 UHFL= OUFL $=$

HIST AO ADE PULE HELGH

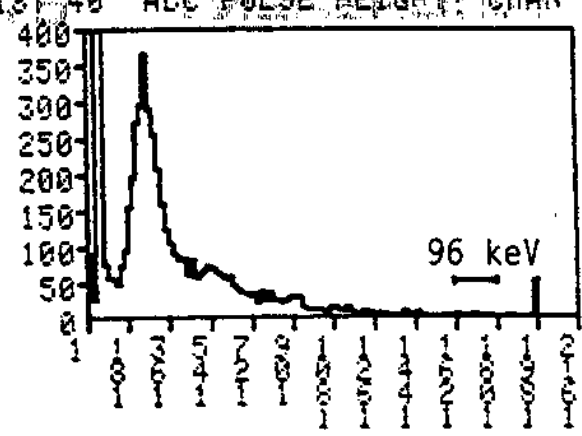

TOTAL $=5970$ BNFL $=0$ OUF $L=9$

Fig. 10 


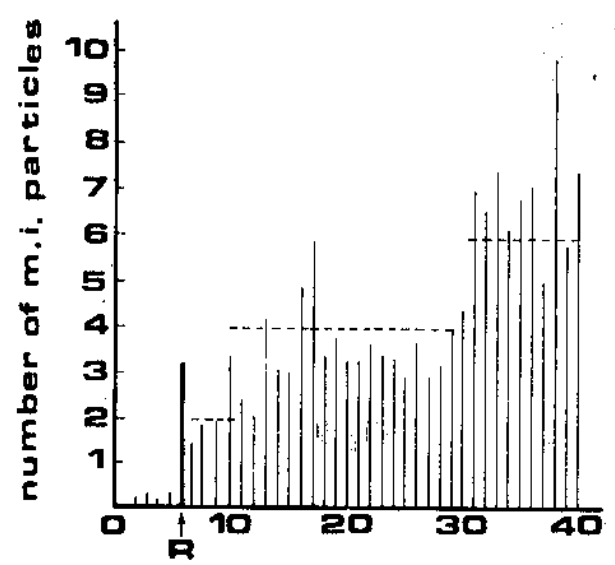

P = nuclear recoils

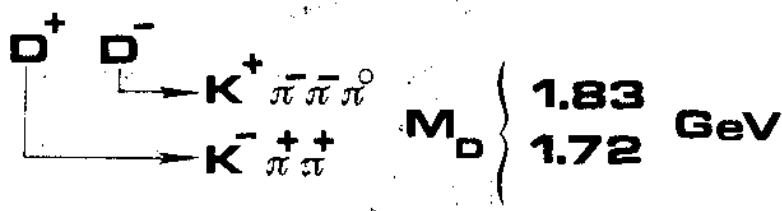

COHERENT

A)
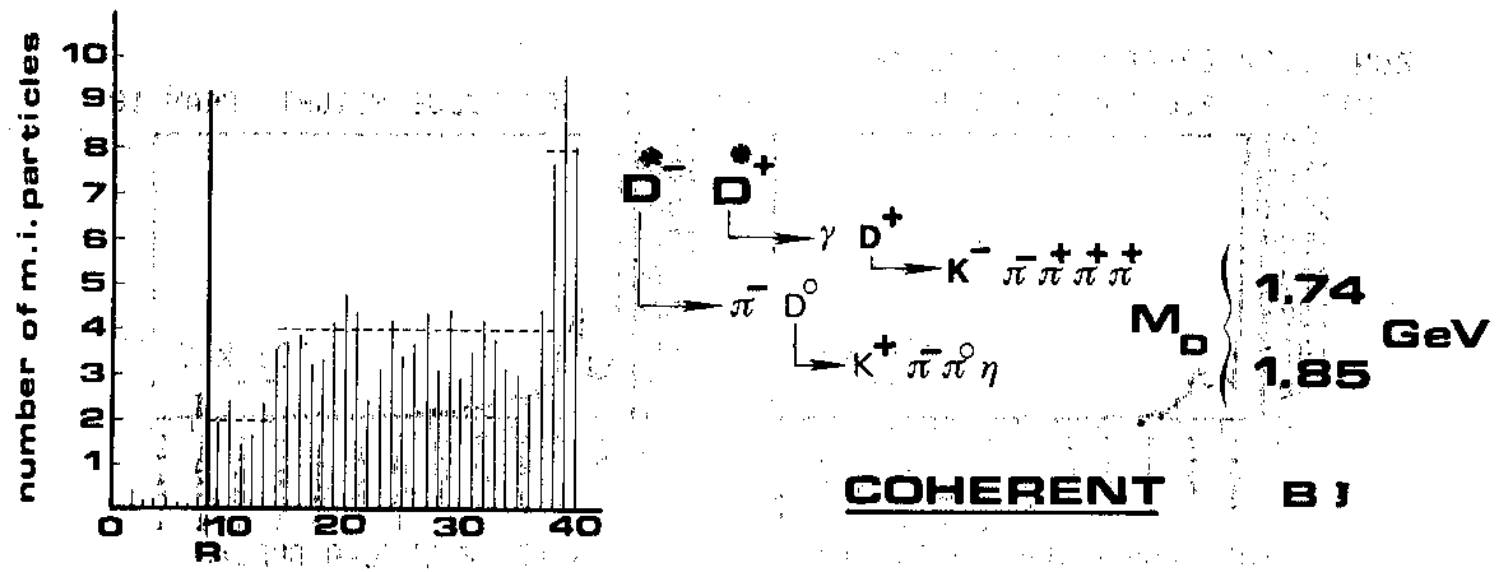

COHERENT B
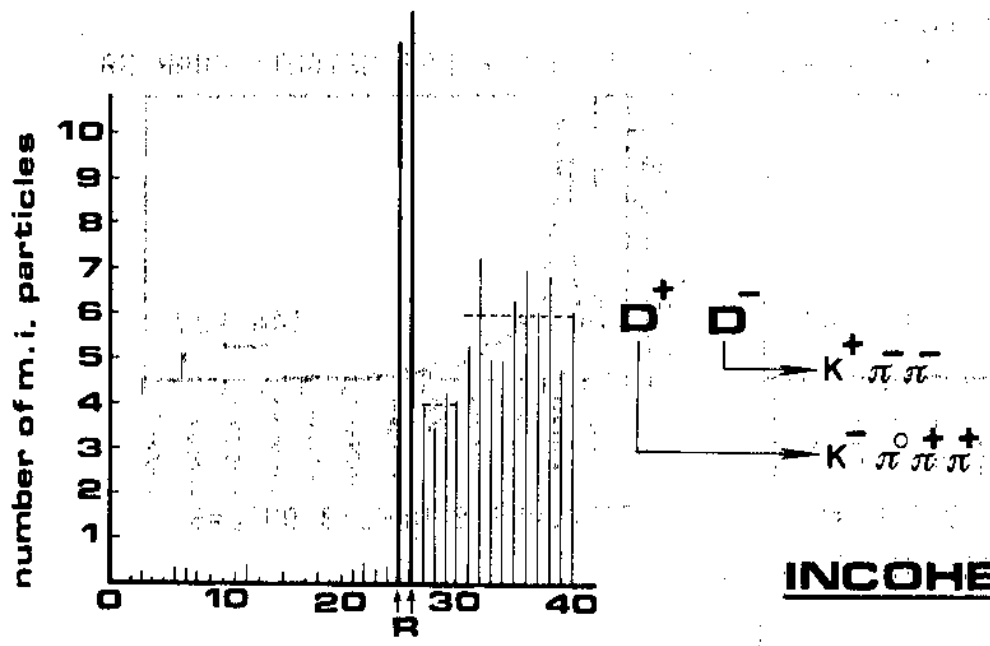

$\mathrm{M}_{0}^{1.72} \mathrm{GeV}$

INCOHERENT

c)

Si detector number

Fig. 11 